Ann. Biol. anim. Bioch. Biophys., 1977, 17 (4), 515-522.

\title{
Mise au point d'une méthode d'enregistrement chronique du débit duodénal chez le mouton : exemple d'application
}

\author{
par C. PONCET, E. DIMOVA, M. LEVEILLÉ*, C. DARDILLAT
}

Station de Recherches sur l'Elevage des Ruminants, I. N. R. A., Theix, Saint Genès-Champanelle, 63110 Beaumont

* 40, rue Anatole France, 63100 Clermont-Ferrand.

Summary. Permanent recording of duodenal flow in sheep.

Feeding behavior, duodenal flow and gastroduodenal motility of adult sheep were recorded simultaneously and permanently.

Movement and flow of duodenal contents were measured on sheep fitted with re-entrant cannulae using an electromagnetic flowmeter. This apparatus was especially designed to measure duodenal flow (Poncet et al., 1976); its characteristics and performance are presented in Table 1.

Feeding behavior data (eating and ruminating time, amplitude and frequency of jaw movements) were recorded by means of a probe using the Doppler effect and placed under the jaw.

Gastroduodenal motility was recorded electromyographically (Ruckebusch, 1970).

Using these three techniques simultaneously, preliminary results obtained from two sheep were compiled. Table 2 shows :

- a modification of gastroduodenal electrical activity after duodenal cannulation ;

- a relationship between motility and flow in the fistulated sheep ;

- an analysis of flow variations determined by feeding behavior, day and animal.

The number of bursts of spike potentials migrating along the entire duodenum decreased (15 and 30 p. 100) after cannulation ; all bursts were accompanied by a flow gush. The frequency of front activity increased 8 to 22 and 15 to $24 / 24 \mathrm{~h}$ below the section ; it became very variable ( 4 to $19 / 24 \mathrm{~h}$ ) above the section. Front activity was rarely synchronous on both sides.

In the same sheep, diurnal variations in flow gush volume were small, but the number of gushes increased during rumination. Between days, flow variations were due to differences in number and/or volume of gushes. Large individual daily flow variations (16.5 I/day and $8.2 \mathrm{l} /$ day) were noted, mainly due to large differences of gush volume, although the number of gushes did not vary.

A partir de la méthode débitmétrique électromagnétique proposée par Singleton (1961) et utilisée récemment (Bueno, Fioramonti et Ruckebusch, 1975 ; Dardillat, 1975) pour détecter le passage des bols alimentaires, nous avons réalisé un débitmètre 
adapté à la mesure quantitative directe du débit duodénal au niveau d'une canule réentrante chez le mouton. Nous présentons ici les caractéristiques de l'appareil ainsi que les possibilités offertes par cette technique d'enregistrement graphique en continu du débit duodénal.

\section{Méthode de mesure du débit duodénal.}

La mesure du débit duodénal est effectuée par débitmétrie électromagnétique sur des moutons munis de canules réentrantes. En période de mesure, une sonde électromagnétique extracorporelle est placée entre les deux canules, laissant l'animal libre de tout mouvement. Pour supprimer les oscillations du contenu digestif dans la canule, provoquées par les mouvements respiratoires, un clapet amortissant les reflux et n'offrant qu'une très faible résistance au flux est placé en amont de la sonde.

Après avoir montré, à l'aide d'un débitmètre sanguin, que cette technique permet de mesurer le débit duodénal avec précision (Poncet et al., 1976) nous avons construit et utilisé un débitmètre spécialement adapté à cette mesure (fig. 1).

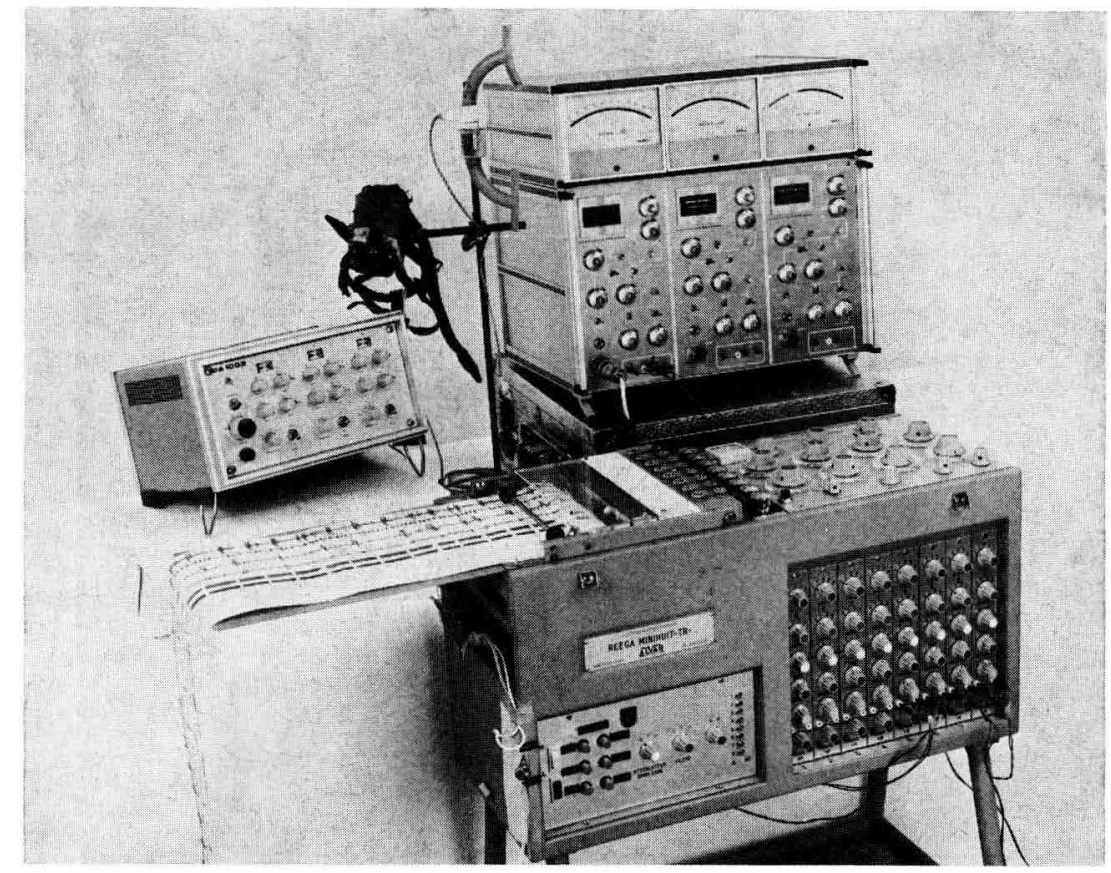

FIG. 1. - Appareils utilisés pour l'enregistrement du comportement alimentaire et de l'électromyogramme gastrointestinal et pour la mesure du débit du contenu duodénal

\section{Caractéristiques de l'appareil.}

Le flux duodénal se caractérisant par un débit intermittent avec flux et reflux, et par l'hétérogénéité du fluide en mouvement (suspension de particules de taille 
Mesure du débit duodénal chez le mouton

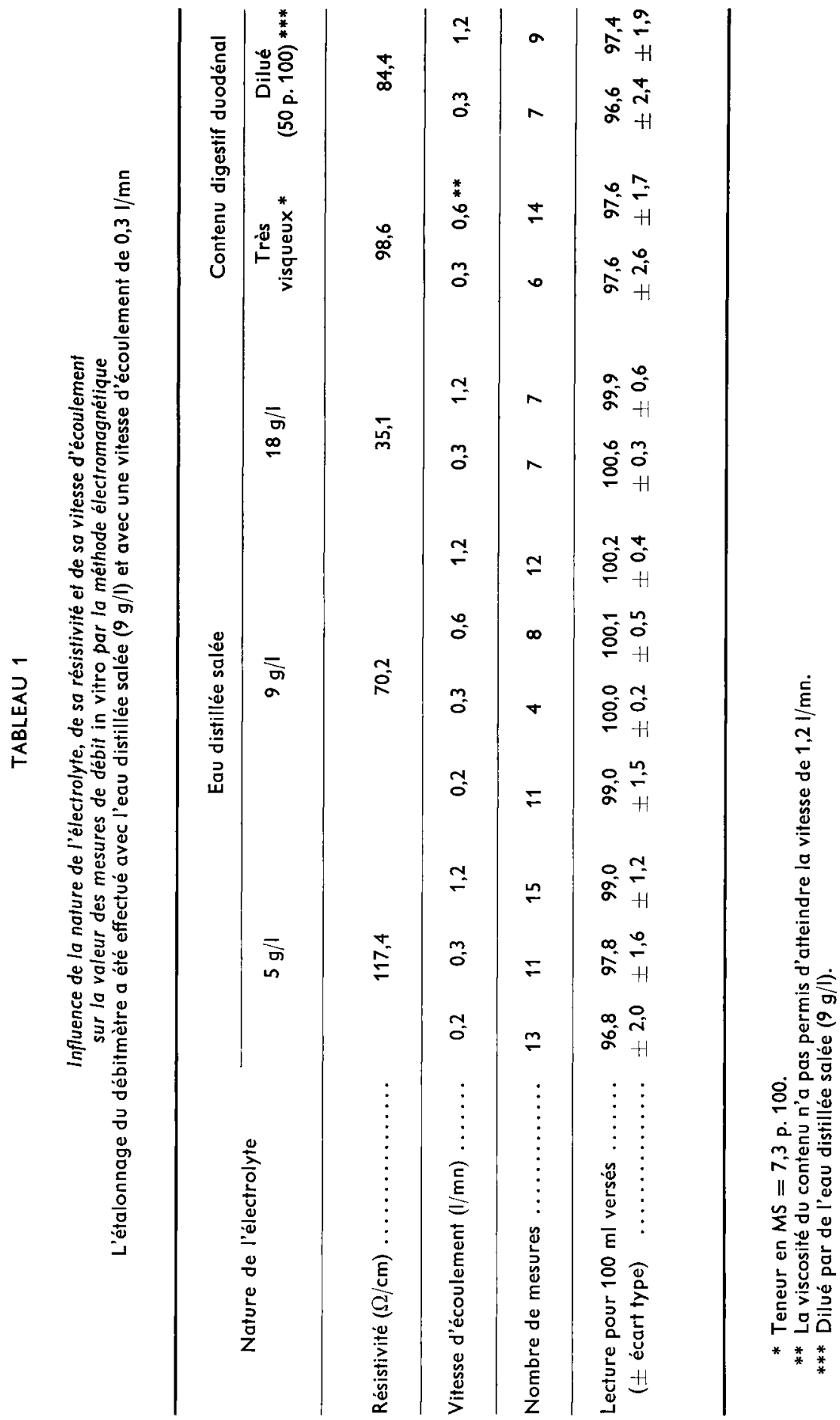


variable dans un liquide conducteur), nous avons dû construire un débitmètre possédant une courbe de réponse linéaire pour une gamme de vitesse étendue $(0 \mathrm{à} 1,5 \mathrm{l} / \mathrm{mn})$, une ligne de base stable en milieu hêtérogène, un intégrateur à comptage ef décomptage.

La principale difficulté a été de limiter l'instabilité de la ligne de base en période d'arrêt du débit. En effet, au cours de ces périodes, les particules du contenu digestif sédimentent. II en résulte une hétérogénéité de répartition des particules dans le champ magnétique qui produit un décalage lent mais important du zéro, enregistré comme un débit. Pour éliminer ces artéfacts, un système d'indexation du seuil de sensibilité de l'appareil aux vitesses de déplacement de la ligne de base a été prévu, éliminant les impulsions électriques qui s'établissent à une vitesse inférieure au seuil choisi. Les informations données en continu par ce débitmètre, soit visuellement, soit graphiquement, sont la vitesse instantanée du débit et le volume de chaque flux et reflux (fig. 2). Un intégrateur digital affiche le volume total.

\section{Essais débitmétriques in vitro (tabl. 1).}

Nous avons testé in vitro les performances de l'appareil dans les conditions suivantes : la sonde électromagnétique et le clapet sont placés à l'une des extrémités d'un tube en $U$ donf l'autre extrémité est munie d'un entonnoir ; l'appareil, réglé à la sensibilité utilisée pour les mesures in vivo est étalonné avec du sérum physiologique, à la vitesse d'écoulement de $0,3 \mathrm{l} / \mathrm{mn} ; 100 \mathrm{ml}$ de différents liquides (eau distillée salée, contenu digestif duodénal) sont versés dans l'entonnoir à une vitesse aussi constante que possible. Nous avons ainsi réalisé des conditions de mesure extrêmes (viłesse d'écoulement variant de 0,2 à $1,21 / \mathrm{mn}$, résistivité du liquide variant de 35 à $120 \Omega / \mathrm{cm}$ ).

L'écart maximum observé entre le volume versé et la mesure est de 3,4 p. 100. L'influence de la nature de l'électrolyte (eau salée ou contenu duodénal) semble prépondérante indépendamment de la résistivité, pour des raisons indéterminées (nature des ions, teneur en matière sèche...). L'influence de la résistivité du liquide sur la précision des mesures est faible ; celle de la vitesse d'écoulement est d'autant plus importante que le liquide a une résistivité plus grande (cas de l'eau salée à $5 \mathrm{~g} / \mathrm{l}$ ). La reproductibilité des mesures est satisfaisante (coefficient de variation maximum : 2,7 p. 100), elle diminue sensiblement lorsque la résistivité du milieu augmente ef lorsque la vitesse diminue.

\section{Exemple d'application.}

La possibilité d'enregistrer le débit duodénal en continu dans des conditions physiologiques nous a permis d'aborder l'étude de l'influence du régime alimentaire sur le transit digestif, la motricité gastroduodénale et le comportement alimentaire, à partir d'enregistrements simultanés de ces 3 paramètres de la digestion chez le mouton.

Nous avons enregistré la motricité gastroduodénale (réseau, fevillet, caillette, 40 premiers centimètres du duodénum) par électromyographie (Ruckebusch, 1970), et le comporiement alimentaire à l'aide de capteurs de mouvements à effet Doppler, 
placés sous la mâchoire inférieure du mouton (fig. 1a). Cette technique nouvelle permet de connaître les temps d'ingestion ef de rumination, ainsi que l'amplitude et la fréquence des mouvements de la mâchoire (fig. 2). Tous les enregistrements ont été recueillis sur un polygraphe (encéphalographe Reega VIII, Alvar).

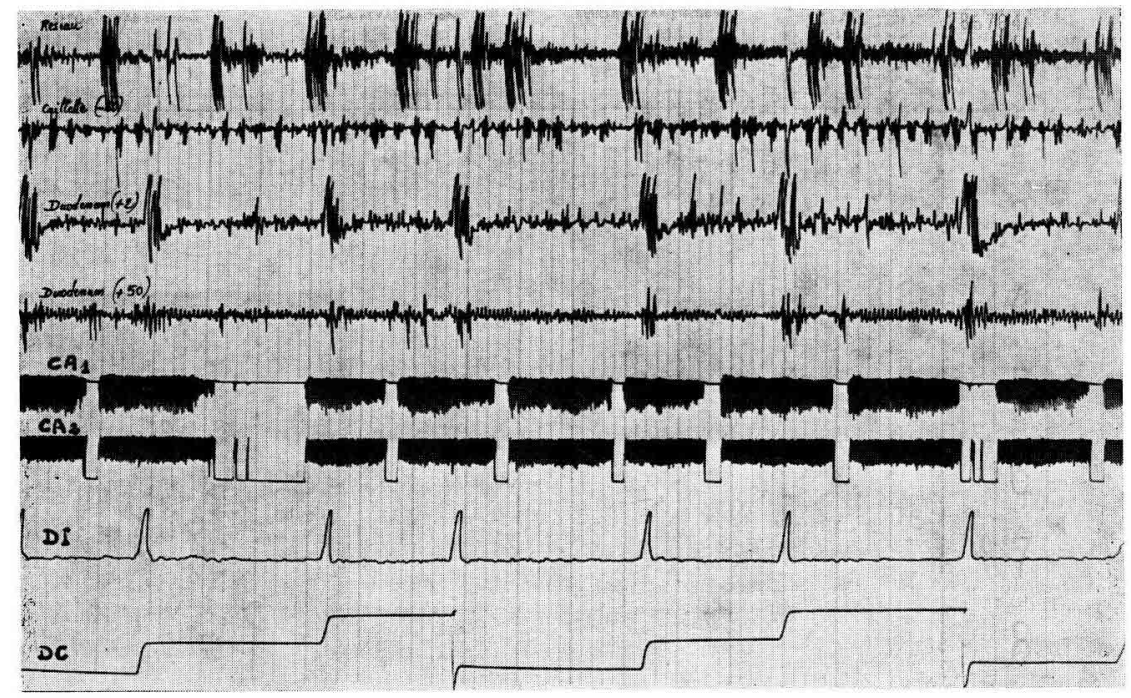

FIG. 2. - Exemple d'enregistrement simultané du comportement alimentaire (amplitude, $\mathrm{CA}_{1}$, et fréquence, $\mathrm{CA}_{2}$ des mouvements de la mâchoire), de l'électromyogramme gastrointestinal (réseau, $\mathrm{R}$; caillette, $C$ partie fondique ; duodénum : $2 \mathrm{~cm}$ du pylore, $D_{1} ; 50 \mathrm{~cm}$ du pylore, $D_{2}$ ) ef du débit duodénal ( $\mathrm{DI}$ : débit instantané ; $\mathrm{DC}$ : débit cumulé).

Nous rapportons ici les premiers résultats (tabl. 2) de l'enregistrement des 3 paramètres étudiés sur 2 moutons adultes, l'un de race Texel ( $43 \mathrm{~kg})$, l'autre de race Limousine $(44 \mathrm{~kg})$, recevant une ration composée de foin et d'aliment concentré $(3: 1)$. Les quantités ingérées ont varié de 1000 à $1200 \mathrm{~g} / \mathrm{j}$ selon l'animal et la période considérée (avant et après la fistulation) en raison de refus de foin. Nous avons enregistré le comportement alimentaire ef l'électromyogramme avant la pose des canules duodénales. Les enregistrements de débit ont eu lieu selon les moutons, du $22^{e}$ au $43^{\mathrm{e}}$ jour ou du $13^{\mathrm{e}}$ au $21^{\mathrm{e}}$ jour après l'opération.

Influence de la section infestinale sur l'électromyogramme : relation entre motricité ef débit.

La pose de la canule réentrante n'a pas modifié le temps d'ingestion de la ration mais a augmenté de 30 et 40 p. 100 le temps de rumination. La motricité du réseau ne semble pas être modifiée avant et après l'opération ( 83 ef 87 contractions par $h$ en moyenne pendant les $7 \mathrm{~h}$ suivant le repas). L'activité du feuillet (grande courbure) est nettement accrue : 84 à 100 contractions (moyenne de 4 jours d'enregistrement), et plus régulièrement répartie au cours de la journée. Cependant, ces deux dernières observations n'ont porté que sur un seul animal et sont à confirmer. 


\section{TABLEAU 2}

Principaux résultats obtenus par enregistrement simultané du comportement alimentaire, de l'électromyogramme duodénal et du débit duodénal sur deux moutons, avant et après la pose de canules duodénales réentrontes

\begin{tabular}{|c|c|c|c|c|}
\hline Période d'enregistrement par rapport à la fistulation & Avant & $\begin{array}{c}\text { Après } \\
\left(22^{\mathrm{e}} \text { au } 43^{\mathrm{e}} \mathrm{j}\right)\end{array}$ & Avant & $\begin{array}{c}\text { Après } \\
\left(13^{\mathrm{e}} \text { au } 21^{\mathrm{e}}\right.\end{array}$ \\
\hline Quantités ingérées $(g \mathrm{MS} / \mathrm{j}) \ldots \ldots \ldots \ldots$ & 1000 & 1100 & 1200 & 1100 \\
\hline \multicolumn{5}{|l|}{ Comportement alimentaire $(\mathrm{mn} / 24 \mathrm{~h})$} \\
\hline 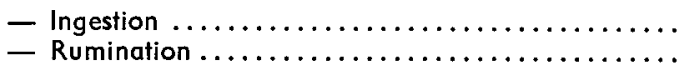 & $\begin{array}{c}127 \\
278(6) *\end{array}$ & $\begin{array}{c}120 \\
369(6)\end{array}$ & $\begin{array}{c}221 \\
343(2)\end{array}$ & $\begin{array}{c}203 \\
496(5)\end{array}$ \\
\hline
\end{tabular}

Electromyogramme duodénal

- Nombre de salves propulsives, $5 \mathrm{~cm}$ après le pylore (moyenne/h) .........................

— Fréquence de l'activité régulière (moyenne/j) .....

$\begin{array}{cccc}43,3(3) * & 31,0(11) & 41,2(3) & 34,9(10) \\ 8(6) & 22(5) & 15(5) & 24(12)\end{array}$

\section{Débit}

- Nombre de jours de mesure ..................

- Débit moyen, $1 / j$..........................

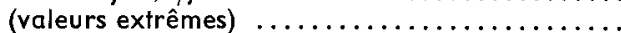

- Nombre de vagues par heure .................

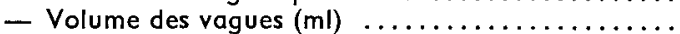

- Débit horaire moyen $(\mathrm{ml})$ :

- pendant l'ingestion ......................

- pendant la rumination ......................

- Nombre de vagues par heure:

- pendant l'ingestion .......................

- pendant la rumination .........................

- Volume des vagues $(\mathrm{ml})$ :

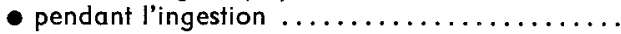

- pendant la rumination .....................

* Nombre de jours de mesure.

$15(5)$

** Ecart-type.

$\begin{array}{cc}6 & 5 \\ 16,5 & 8,2 \\ (14,1-18,7) & (7,1-9,7) \\ 31,4 \pm 3,9 * * & 33,9 \pm 2,3 \\ 22,6 \pm 3,7 & 10,1 \pm 0,9 \\ 695 \pm 78 & 300 \pm 44 \\ 734 \pm 92 & 392 \pm 60 \\ 27,4 \pm 3,5 & 30,1 \pm 3,9 \\ 34,2 \pm 3,5 & 37,3 \pm 2,3 \\ 25,6 \pm 3,3 & 10,1 \pm 0,8 \\ 21,7 \pm 4,0 & 10,5 \pm 0,9 \\ & \end{array}$

Au niveau duodénal, la fistulation avec section intestinale transversale, $15 \mathrm{~cm}$ en aval du pylore, a eu des conséquences importantes sur l'activité myoélectrique :

- Nombre de salves de potentiel (ou contractions) composant l'activité irrégulière.

Avant l'opération, nous avons compté, $5 \mathrm{~cm}$ après le pylore, 50 salves de potentiels/h (moyenne de 4 jours) dont 41 propagées sur tout le duodénum, donc probablement propulsives. Après l'opération, le nombre de salves propulsives (comptées sur 10 jours) est égal au nombre de vagues de débit et a diminué de 15 et 30 p. 100 par rapport à sa valeur antérieure. 
- Fréquence des phases d'activité régulière ef synchronisation des solves de potentiel après l'opération.

Avant la fistulation, la fréquence des phases d'activité segmentaire a été très différente entre les deux moutons utilisés : 8 et 15 par 24 h (moyenne de 6 jours de mesure). La propagation de ces activités suivies de périodes de repos de 10 à $15 \mathrm{mn}$ était cependant normale.

Après l'opération, la fréquence des activités régulières en aval de la section s'est fortement accrue, tandis qu'en amont, elle est devenue très variable : 4 à 19 par $24 \mathrm{~h}$. L'activité régulière se propage rarement à partir du pylore ; elle prend naissance après la section, et, lorsqu'elle existe avant celle-ci, elle apparaît après le départ de l'activité régulière sur le duodénum distal, avec un décalage de 5 à $15 \mathrm{mn}$, sous une forme souvent peu différenciée, provoquant une agitation du contenu dans la canule et parfois des débits. On peut reconnaître, en amont et en aval de la section, les trois phases de l'activité myoélectrique intestinale; mais elles ne sont pas synchrones de part ef d'autre. Ainsi, l'arrivée de salves propulsives ef de débits en provenance du duodénum en amont de la section peut perturber les phases de repos sur le duodénum distal. Cependant, sur les courbes représentant l'évolution du débił par période de $10 \mathrm{mn}$, les périodes d'arrêt de débit ou de débit minimum correspondent toujours au départ d'une activité régulière.

- Variations du débit en relation avec le comportement alimentaire.

Pendant les périodes de rumination, le débit duodénal est généralement plus élevé que pendant les périodes d'ingestion. Ces différences s'expliquent principalement par la variation du nombre des vagues de contenu digestif $(27,4 \pm 3,5$ et $30,1 \pm$ 5,9 vagues par h pendant l'ingestion ; 34,2 $\pm 3,5$ et $37,3 \pm 2,3$ pendant la rumination) dont le volume est peu variable au cours du nycthémère. Le débit du contenu duodénal varie également selon le jour de prélèvement et selon l'animal $(16,5 \mathrm{l} / \mathrm{jour}$ et $8,2 \mathrm{l} / \mathrm{jour}$ pour les moutons que nous avons utilisés). Cette différence est due à une différence du volume des vagues de contenu digestif $(22,6 \pm 3,7 \mathrm{ml}$ contre $10,1 \pm 0,9)$ peut être liée au génolype différent des deux animaux (Texel, Limousin). En revanche, le nombre de vagues n'a pas varié $(31,4 \pm 3,9$ vagues/h contre $33,9 \pm 2,3)$.

\section{Conclusion.}

La débitmétrie électromagnétique est une technique précise, permettant de mesurer directement le débit duodénal pendant de longues périodes, sans inconvénient pour l'animal. Elle rend possible l'enregistrement simultané du comportement alimentaire, de la motricité et du débit duodénal qui sont trois paramètres de la digestion étroitement liés. Cependant, elle nécessite actuellement la pose de canules réentrantes dont l'effet sur le transit digestif est mal connu, mais semble important, comme en témoignent les modifications de l'électromyogramme gastroduodénal. Les résultats que nous avons obtenus ne permettent pas de savoir si ces modifications sont permanentes ou si elles disparaissent avec le temps, et quelle est leur incidence 
sur le transit digestif. L'utilisation d'une sonde éleciromagnétique intracorporelle sans section intestinale devrait permettre de résoudre ces différents problèmes.

Commission CNERNA Digestion-Absorption, Tours, 13 novembre 1976.

\section{Références}

BUENO L., FIORAMONTI J., RUCKEBUSCH Y., 1975. Rate of flow of digesta and electrical activity of the small intestine in dogs and sheep. J. Physiol., 249, 69-85.

DARDILLAT C., 1975. In RUTTER J. M. Perinatal ill-health in calves, 111-122, Simpsons Press, Newbury, Berkshire, U. K.

PONCET C., DIMOVA H., DARDILLAT C., THIVEND P., 1976. Measurement of flow of intestinal contents in sheep by an electromagnetic method. Proc. Nutr. Soc., 35, $107 \mathrm{~A}$.

RUCKEBUSCH Y., 1970. The electrical activity of the digestive tract of the sheep as an indication of the mechanical events in various regions. J. Physiol., 210, 857-882.

SINGLETON A. G., 1961. The electromagnetic measurement of the flow of digesta through the duodenum of the goat and the sheep. J. Physiol., 155, 134-147. 